\title{
POBLACIONES E IDENTIFICACIÓN DE LOS HONGOS CAUSANTES DE MOHOS POSCOSECHA EN EL PEDÚNCULO DE LA PIÑA, EN DOS ZONAS DE COSTA RICA
}

\author{
Johanny Castro Chinchilla ${ }^{1 *}$, Gerardina Umaña Rojas* \\ Palabras clave: Ananas comosus; Penicillium; mohos; fases poscosecha; ceras. \\ Keywords: Ananas comosus; Penicillium; molds; postharvest phases; waxes.
}

Recibido: 27/03/15

\section{RESUMEN}

El desarrollo de mohos en el pedúnculo de la piña de Costa Rica, es uno de los problemas poscosecha más importantes que conlleva al rechazo de la fruta en el mercado destino. El objetivo de esta investigación fue evaluar las poblaciones de los hongos causantes de mohos en el pedúnculo de la piña, en diferentes fases de procesamiento poscosecha, mediante muestreos mensuales durante un año de producción en 2 zonas. Además, se realizó la identificación morfológica y molecular de los hongos más frecuentes. Se cuantificó, in vitro, el número de unidades formadoras de colonias (UFC) en el agua de desinfección, la cera, el aire de las cámaras de almacenamiento y en el pedúnculo y la cáscara de la fruta, sin procesar (SP) y procesada $(\mathrm{P})$ con los tratamientos comerciales de las fincas. Además, se evaluó la incidencia y severidad de moho en el pedúnculo. Hubo variaciones en las poblaciones de hongos en todas las fases de procesamiento y en la fruta, con valores más altos en la cera que en el agua de desinfección. En el pedúnculo de fruta SP se obtuvo poblaciones más altas que en fruta $\mathrm{P}$, lo cual coincidió con mayores porcentajes de moho al finalizar el almacenamiento. En las cámaras de enfriamiento también se recuperó esporas de hongos, que podrían ser una fuente

Autor para correspondencia. Correo electrónico: johanny.castrochinchilla@ucr.ac.cr
Aceptado: 10/06/15

\begin{abstract}
Populations and identification of fungi causing postharvest molds, on pineapple peduncles in two regions in Costa Rica. Pineapple peduncle mold is an important postharvest problem in Costa Rica and it causes fruit rejection. The objective of this study was to identify and quantify the most important fungi in different postharvest phases. Monthly samplings were performed during one production year in 2 regions of Costa Rica. The main genera of fungi were identified and characterized at the molecular level. The colony forming units (CFU) were determined in disinfection water, wax, cooling rooms air and in the peel and peduncle of fruits before (NP) and after (P) processing with the common postharvest treatments of the farms. Fruits were stored in cooling rooms during 22 days and at the end incidence and severity of peduncle molds were evaluated. During the year, changes in fungi populations were observed in all postharvest phases and in the fruits, with higher populations in wax than in disinfection water. Fungi population and molds were higher in the peduncle of NP fruits as compared with P fruits, coincident with larger mold populations at the end of storage. Fungi recovered in the cooling rooms air could also be a source for peduncle
\end{abstract}

Centro de Investigaciones Agronómicas, Universidad de Costa Rica. San José, Costa Rica. 
de inóculo para el desarrollo de moho. Los principales hongos identificados fueron Penicillium purpureogenum, $P$. diversum y Penicillium sp., los cuales presentaron alta esporulación in vitro y crecimiento en el pedúnculo. Además en diferentes puntos del proceso como el encerado y el enfriamiento, en los que se capturaron esporas, la fruta podría ser contaminada, por lo que se considera importante la adecuada limpieza de las cámaras de enfriamiento, así como evitar que la cera acumule poblaciones importantes de microorganismos.

\section{INTRODUCCIÓN}

El cultivo de piña (Ananas comosus L.) como monocultivo a gran escala se inició a finales de 1970 en el sur de Costa Rica y una de las variedades más utilizada ha sido la Dorada Extradulce. Esta actividad se expandió rápidamente durante los últimos 10 años en el Caribe y el Norte del país, alcanzando un área de aproximadamente 43000 a 45000 has y con una exportación a Europa y Estados Unidos de 2095702 toneladas, lo cual generó en el 2014, un ingreso para el país de $\$ 874,1$ millones (CANAPEP 2015, PROCOMER 2015).

El desarrollo de moho en el pedúnculo $\mathrm{u}$ otros patógenos que afectan el fruto de piña, causa pérdidas importantes para los productores, debido al costo económico de los reclamos por parte de los clientes y la devolución de los contenedores (Wills et ál. 1998, Barquero 2010). En la mayoría de las ocasiones, los síntomas debido a este tipo de daño no son visibles al momento de la cosecha y se desarrollan cuando la fruta se encuentra en el mercado destino (Dennis 1983, Wills et ál. 1998).

En trabajos realizados en Costa Rica por Barquero (2010), López (2012) y Reyes (2012), se recuperó en el pedúnculo de la piña, los hongos de los géneros Penicillium, Fusarium, Aspergillus y molds development. Penicillium purpureogenum, $P$. diversum and Penicllium sp., were the main fungi identified, with an in vitro high sporulation rate and growing in the peduncle. Moreover, different commercial practices, such as waxing and cooling, where spores were captured, can enhance the peduncle molds development, so it is considered important the cleaning of cooling rooms, as well as developing mechanisms to avoid accumulation in wax of important populations of microorganisms.

Trichoderma. Así mismo, la especie Penicillium funiculosum fue el principal hongo aislado en frutos de piña producidos en Costa Rica y procesados como fruto precortado en España, donde se estudió la influencia de las condiciones de empaque sobre la vida de anaquel bajo condiciones de almacenamiento de $5^{\circ} \mathrm{C}$ y rangos de oxígeno de 11 a 40\% (Montero et ál. 2008). En Hawaii, se trabajó en la búsqueda de organismos para el control biológico del hongo Thielaviopsis paradoxa en frutos de piña y se encontró los géneros Acremonium, Cephalosporium, Cladosporium, Fusarium, Geotrichum, Gliocladium y Penicillium como hongos presentes comúnmente en el fruto y que podrían estar relacionados con el desarrollo de mohos en el pedúnculo (Reyes 1999).

En cultivos como manzana, se ha encontrado que la contaminación de la fruta con diferentes especies del género Penicillium que causan la pudrición de la misma, se da principalmente durante el manejo poscosecha, y se indica que con una densidad de aproximadamente 2500 esporas. $\mathrm{m}^{-3}$, se puede presentar el desarrollo de pudriciones, principalmente si la fruta se almacena bajo condiciones de alta humedad relativa (Amiri y Bompeix 2005). En la investigación realizada por estos autores, la mayor densidad de esporas en la superficie de la fruta coincidió con la mayor densidad de esporas en la atmósfera 
de las cámaras de almacenamiento, con valores entre 4000 y 5000 esporas. $\mathrm{m}^{-3}$.

Debido a que el desarrollo de mohos en poscosecha es uno de los principales problemas en la producción de piña en Costa Rica, se planteó la presente investigación, con el objetivo de evaluar las poblaciones e identificar los hongos causantes de mohos en el pedúnculo de frutos de piña durante diferentes fases de procesamiento poscosecha y determinar durante un año de producción, si existe diferencia entre las poblaciones de estos microorganismos en fruta procesada y sin procesar, en 2 zonas de Costa Rica.

\section{MATERIALES Y MÉTODOS}

El trabajo de investigación se llevó a cabo en 2 zonas productoras de piña en Costa Rica: cantón de Puntarenas en la provincia de Puntarenas y cantón de Sarapiquí en la provincia de Heredia. En cada zona se seleccionó una finca y se realizó un muestreo mensual de abril de 2012 a marzo de 2013. Ambas fincas las administraba la misma compañía y el manejo poscosecha de la fruta fue semejante durante el desarrollo del trabajo. En cada una, se seleccionó las siguientes fases del procesamiento de la fruta: recibo, lavado, encerado, empaque y enfriamiento.

El manejo poscosecha de la fruta en ambas fincas consistió en un lavado de la fruta recién cosechada en agua con cloro a una concentración entre 150 y 180 ppm, aplicación sobre la fruta de una mezcla de ceras compuestas por aceite de origen vegetal y ácidos grasos del glicerol y el sorbitan, aplicación de fungicida sobre el pedúnculo y empaque en cajas de cartón corrugado de aproximadamente $12 \mathrm{~kg}$. La periodicidad del cambio del agua en las pilas de lavado fue en promedio de 3 días en Sarapiquí y 6 días en Puntarenas y en cada finca se contó con un programa mensual de limpieza y desinfección de las cámaras de enfriamiento.

En cada finca, se seleccionó para la toma de muestras y frutas, las siguientes fases del procesamiento de la fruta: recibo, lavado, encerado, empaque y enfriamiento.

\section{Lavado y encerado}

A partir del inicio del proceso de empaque, se tomó 6 submuestras de $250 \mathrm{ml}$ de agua en distintos puntos de la pila de lavado y desinfección de la fruta, distribuidos de forma equidistante, los cuales formaron una muestra compuesta, para un total de 15 muestras compuestas, con un intervalo de 15 minutos entre cada una.

En el punto de caída de la cera que se aplica sobre la fruta, se tomó 6 submuestras de 250 $\mathrm{ml}$ de la cera para formar al igual que en el agua una muestra compuesta de 1,51 , a partir de la cual se tomó un volumen de $40 \mathrm{ml}$ y se colocó en un tubo plástico estéril. Estas se tomaron simultáneamente con las del agua de las pilas de lavado, se colocaron en una hielera a una temperatura entre $7^{\circ} \mathrm{C}$ y $10^{\circ} \mathrm{C}$ y se trasladaron al Laboratorio de Tecnología Poscosecha de la Universidad de Costa Rica, donde se mantuvieron en una cámara de incubación a $7{ }^{\circ} \mathrm{C}$ previo a su procesamiento.

Para el aislamiento de los hongos a partir de la cera, se utilizó el método de dilución seriada estándar con 3 diluciones $\left(10^{-3}\right)$ (French y Hebert 1980, Tortora et ál. 2007) y para las muestras de agua no se realizó ninguna dilución. A partir de la dilución final de cada muestra de cera, y de las muestras de agua, se tomó $100 \mu \mathrm{l}$ y se distribuyeron uniformemente con ayuda de una asa Digralsky, en una placa Petri con el medio de cultivo papa dextrosa agar con ácido láctico (PDA $+\mathrm{Al})$ y se incubó a una temperatura de $22^{\circ} \mathrm{C}$ durante 5 días. Posteriormente, se contó el número de unidades formadoras de colonias (UFC) de hongos, para obtener el número de UFC. $\mathrm{ml}^{-1} \mathrm{de}$ la muestra original.

\section{Recibo de fruta y empaque}

En el área de recibo de fruta, se seleccionó una carreta con fruta recién cosechada y con calidad de exportación (color grado 0,5 a 1, calibre 7, sin heridas visibles, pudriciones y sin defectos en la corona como coronas dobles o deformadas), de la que se tomó al azar un total de 28 frutas previo al inicio del procesamiento en la empacadora y 28 frutas procesadas con los tratamientos de lavado, 
selección, encerado y aplicación de fungicida y listas para empaque, en adelante designadas como fruta sin procesar (SP) y fruta procesada $(\mathrm{P})$, respectivamente. Ambos grupos de frutas provenientes de la misma carreta, se colocaron en cajas plásticas o de cartón, se cubrieron con papel kraft y fueron trasladadas al Laboratorio de Tecnología Poscosecha de la Universidad de Costa Rica, donde se almacenaron en cámaras de enfriamiento bajo condiciones que simulan el manejo comercial: 4 días a $7^{\circ} \mathrm{C}, 1$ hora a $18^{\circ} \mathrm{C}$, 14 días a $7^{\circ} \mathrm{C}$ y 3 días a $18^{\circ} \mathrm{C}$. Al finalizar este período, se evaluó en el pedúnculo la incidencia y se estimó la severidad del moho como porcentaje de cobertura de ese tejido.

Tanto de la fruta sin procesar como de la procesada, se seleccionó al azar 5 frutas en el momento de ingreso al laboratorio y 5 frutas al finalizar el período de almacenamiento. A cada fruta se le extrajo un segmento superficial de aproximadamente $5 \mathrm{~cm}^{2}$ de pedúnculo y de cáscara. Cada segmento se colocó en un beaker con $30 \mathrm{ml}$ de agua destilada estéril con una gota de Tween 20 y se agitó durante 5 minutos. A partir de esta suspensión, se utilizó el método de dilución seriada estándar con 2 diluciones $\left(10^{-2}\right)$ para la fruta recién cosechada y 3 diluciones $\left(10^{-3}\right)$ para la fruta almacenada. A partir de la última dilución se tomó $100 \mu \mathrm{l}$ y se colocaron en una placa Petri con el medio PDA + Al, se distribuyeron uniformemente sobre la misma con ayuda de una asa Digralsky y a los 5 días de incubación a $22^{\circ} \mathrm{C}$, se contó el número de UFC por placa de hongos, para obtener el número de UFC. $\mathrm{ml}^{-1}$ de la suspensión inicial.

\section{Enfriamiento y almacenamiento}

En las cámaras de enfriamiento y almacenamiento, se seleccionaron 15 sitios y en cada uno se colocó una placa Petri con PDA + Al, la cual se expuso en ese ambiente durante 15 min, de acuerdo con la metodología utilizada por Buttner y Stetzenbach (1993) y recomendada por Pitt y Hocking (2009), para el muestreo por deposición de esporas de hongos presentes en el aire. Las muestras se colocaron en una hielera a una temperatura entre $7^{\circ} \mathrm{C}$ y $10^{\circ} \mathrm{C}$ y se transportaron al Laboratorio, se dejaron a la temperatura ambiente durante 5 días y una vez transcurrido ese tiempo, se evaluó el número de UFC por placa de hongos.

\section{Identificación de los microorganismos}

Una vez finalizado el conteo de UFC en cada muestra, se aisló en medio de cultivo PDA + $\mathrm{Al}$, los microorganismos presentes y se identificó los más frecuentes mediante la observación en el microscopio y la ayuda de claves de identificación (Barnett y Hunter 1972, von Arx 1974, Ellis 1976, Watanabe 1994, Seifert et ál. 2011). Además, se seleccionó los 6 hongos más frecuentes para la caracterización e identificación molecular en el Laboratorio de Técnicas Moleculares del Centro de Investigaciones en Protección de Cultivos y otro grupo de 5 hongos fue caracterizado en Laboratorio de Biotecnología de Plantas del Centro de Investigaciones Agronómicas. En ambos casos, se analizó las regiones de los espaciadores internos transcritos ITS1 e ITS2 (ITS-Internal transcribed spacers, por sus siglas en inglés), con los imprimadores ITS5/ITS4, de acuerdo con los protocolos específicos de cada Laboratorio de la Universidad de Costa Rica.

\section{Análisis estadístico}

Los datos de las poblaciones de hongos fueron transformados a $\log _{10}(x+1)$, para facilitar la visualización de las tendencias de las mismas durante el año de estudio.

En el análisis estadístico, se incluyó el total de datos de los 12 muestreos para cada fase de la empacadora muestreada por finca. Se utilizó una prueba de F para comparar las varianzas de las poblaciones de hongos, de acuerdo con la siguiente agrupación: pedúnculo $\mathrm{P}$ vs pedúnculo $\mathrm{SP}$ y cáscara $\mathrm{P}$ vs cáscara $\mathrm{SP}$, en fruta recién cosechada y luego de 22 días de almacenamiento, agua vs cera, y porcentaje de moho en fruta procesada vs sin procesar. Una vez comparadas las varianzas, se aplicó una prueba de $\mathrm{t}$ de Student para muestras independientes, suponiendo varianzas 
iguales o desiguales para comparar las medias de acuerdo con la misma agrupación utilizada para la prueba de $\mathrm{F}$.

\section{RESULTADOS}

En la Figura 1 se observa que, las poblaciones de hongos en la cera de ambas zonas fueron mayores que en agua en la mayoría de los muestreos y los valores más altos en cera se alcanzaron en junio y marzo en la zona de Puntarenas y en agosto y noviembre en Sarapiquí. Se destaca en ambas fincas, que en la mayoría de los muestreos no se obtuvo crecimiento de hongos en el agua de las pilas de lavado-desinfección.

Al comparar ambas zonas se observa que, en Sarapiquí las poblaciones de hongos en la cera de julio a diciembre de 2012 tendieron a ser mayores que en Puntarenas.
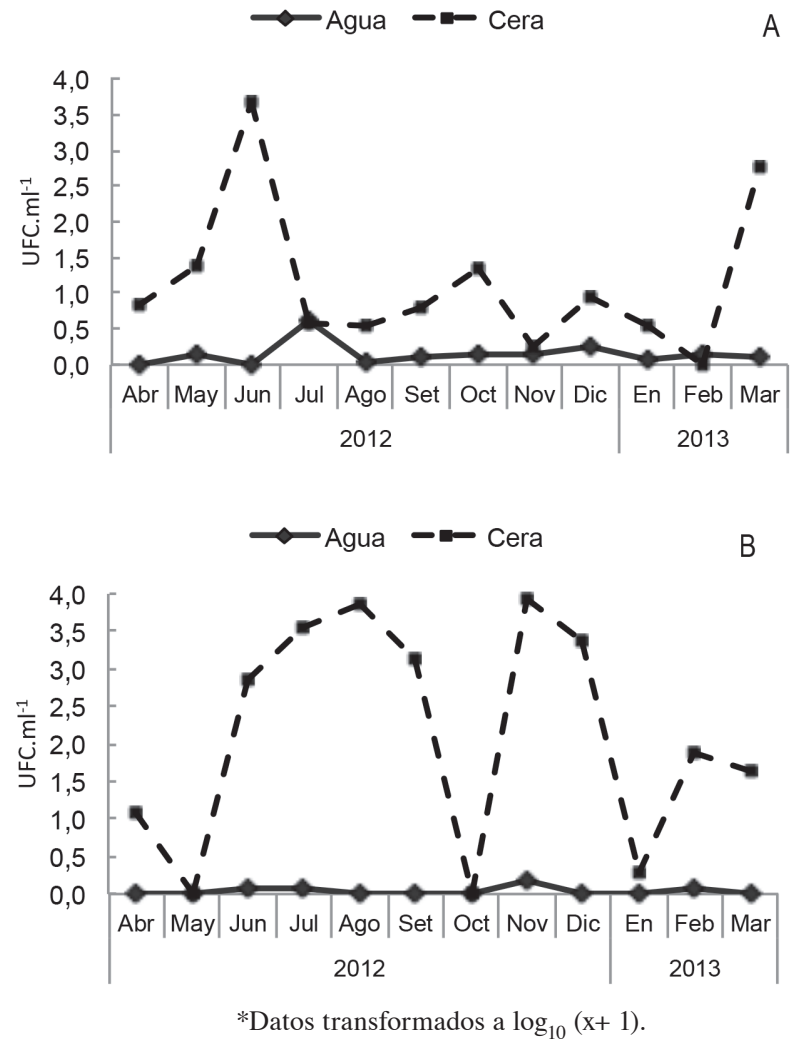

Fig. 1. Promedio mensual de UFC. $\mathrm{ml}^{-1}$ de hongos en aguas de lavado-desinfección y cera de las fincas de Puntarenas (A) y Sarapiquí (B).

Las poblaciones de hongos en el aire de las cámaras de enfriamiento, fueron mayores durante todo el intervalo de evaluación en la zona de Sarapiquí que en Puntarenas, alcanzándose los valores más altos en junio, agosto y marzo (Figura 2).
Las poblaciones de hongos en el pedúnculo al momento de la cosecha en fruta procesada y sin procesar presentaron variaciones durante el año, con valores desde 0 hasta 1,5 UFC. $\mathrm{ml}^{-1}$ en Puntarenas y entre 0 y 2 UFC.ml ${ }^{-1}$ en Sarapiquí, como se muestra en 


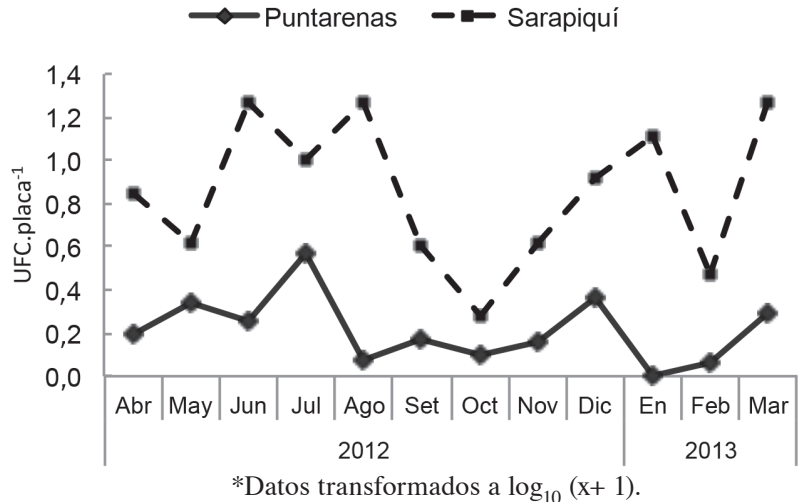

Fig. 2. Promedio mensual de UFC.placa Petri ${ }^{-1}$ de hongos con PDA + Al, en muestreos del aire de las cámaras de enfriamiento.

la Figura 3. A pesar de no observarse una tendencia en el comportamiento de estas poblaciones a lo largo del año evaluado, en la mayoría de los muestreos, la población en fruta sin procesar fue mayor que en fruta procesada (Figura 3).
Las poblaciones de hongos en ambas zonas luego de 22 días de almacenamiento de fruta procesada y sin procesar (Figura 4) fueron mayores que las presentes al momento de la cosecha (Figura 3), con valores registrados desde 0,7 hasta 5,5 UFC. $\mathrm{ml}^{-1}$.
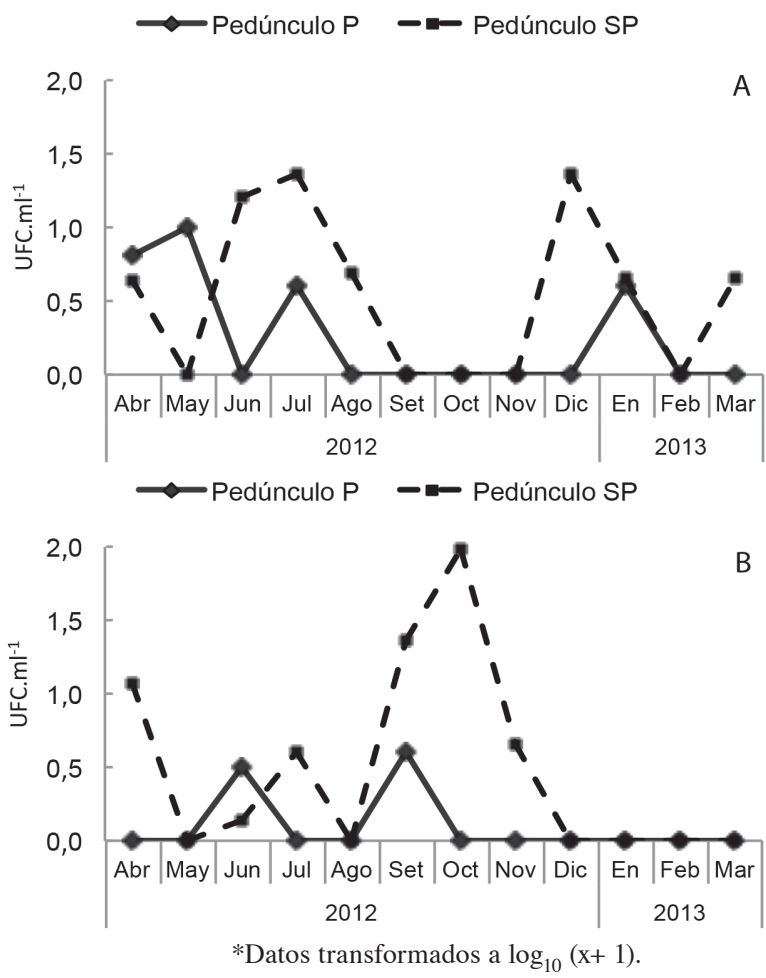

Fig. 3. Promedio mensual de UFC. $\mathrm{ml}^{-1}$ de hongos de la suspensión de pedúnculo de fruta recién cosechada, procesada (P) y sin procesar (SP), de las fincas de Puntarenas (A) y Sarapiquí (B). 

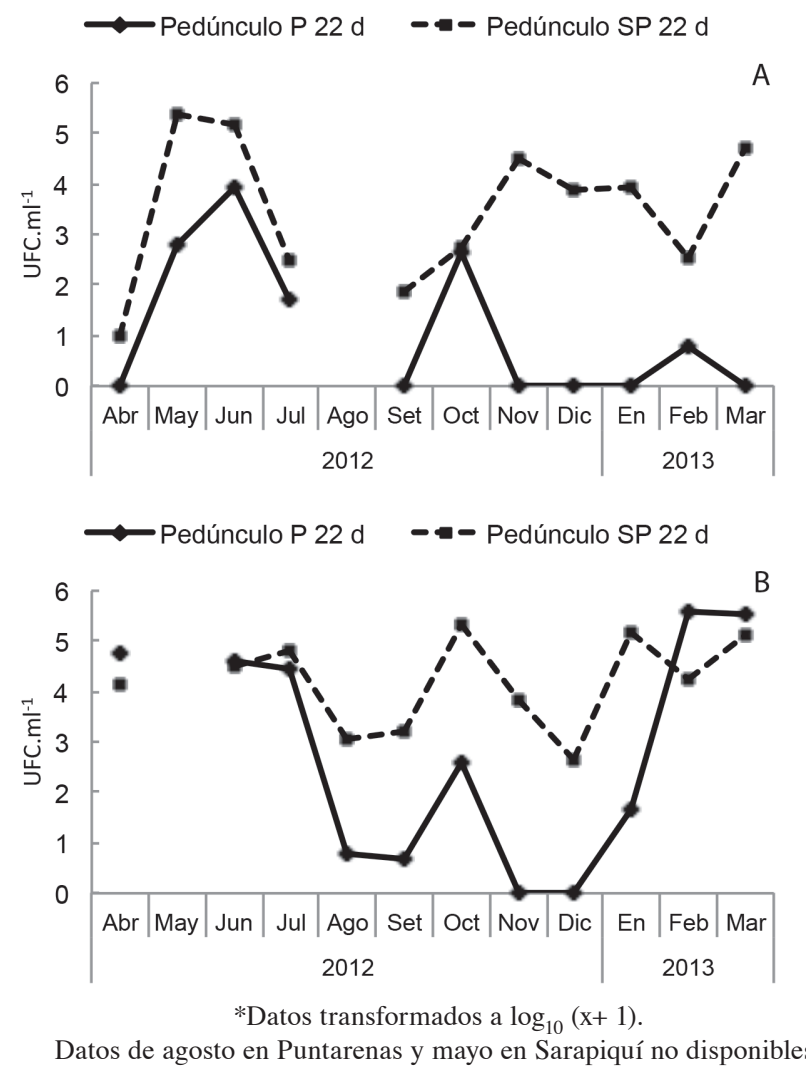

Fig. 4. Promedio mensual de UFC.ml-1 de hongos de la suspensión de pedúnculo de fruta procesada (P) y sin procesar (SP) de las fincas de Puntarenas (A) y Sarapiquí (B), después de 22 días de almacenamiento.

Luego de 22 días de almacenamiento (Figura 4), las poblaciones de hongos en la fruta de ambas zonas fueron mayores que las presentes al momento de la cosecha (Figura 3). Asimismo, como se muestra en la Figura 4, en la mayoría de los muestreos, las poblaciones en el pedúnculo al finalizar el almacenamiento, tendieron a ser mayores en fruta sin procesar que en la procesada, con excepción de octubre de 2012 en Puntarenas y abril y junio de 2012 y febrero y marzo de 2013 en Sarapiquí.

$\mathrm{Al}$ igual que al momento de la cosecha, no se observó una tendencia en las poblaciones de hongos presentes en el pedúnculo durante el año, sin embargo, en la zona de Sarapiquí, se presentaron los valores más altos de UFC. $\mathrm{ml}^{-1}$ de hongo en fruta procesada en abril, junio y julio de 2012, y en febrero y marzo de 2013, y en Puntarenas de mayo a junio y octubre de 2012 .

En la Figura 5 se muestra una población de hongos similar en la cáscara de fruta recién cosechada, procesada y sin procesar de ambas zonas con excepción de enero de 2013 en Puntarenas donde la población en fruta procesada fue menor que en fruta sin procesar y en mayo de 2012 en Sarapiquí, donde no se recuperó hongos en la cáscara de fruta procesada y en la fruta sin procesar se obtuvo 3,5 UFC.ml- ${ }^{-1}$. 

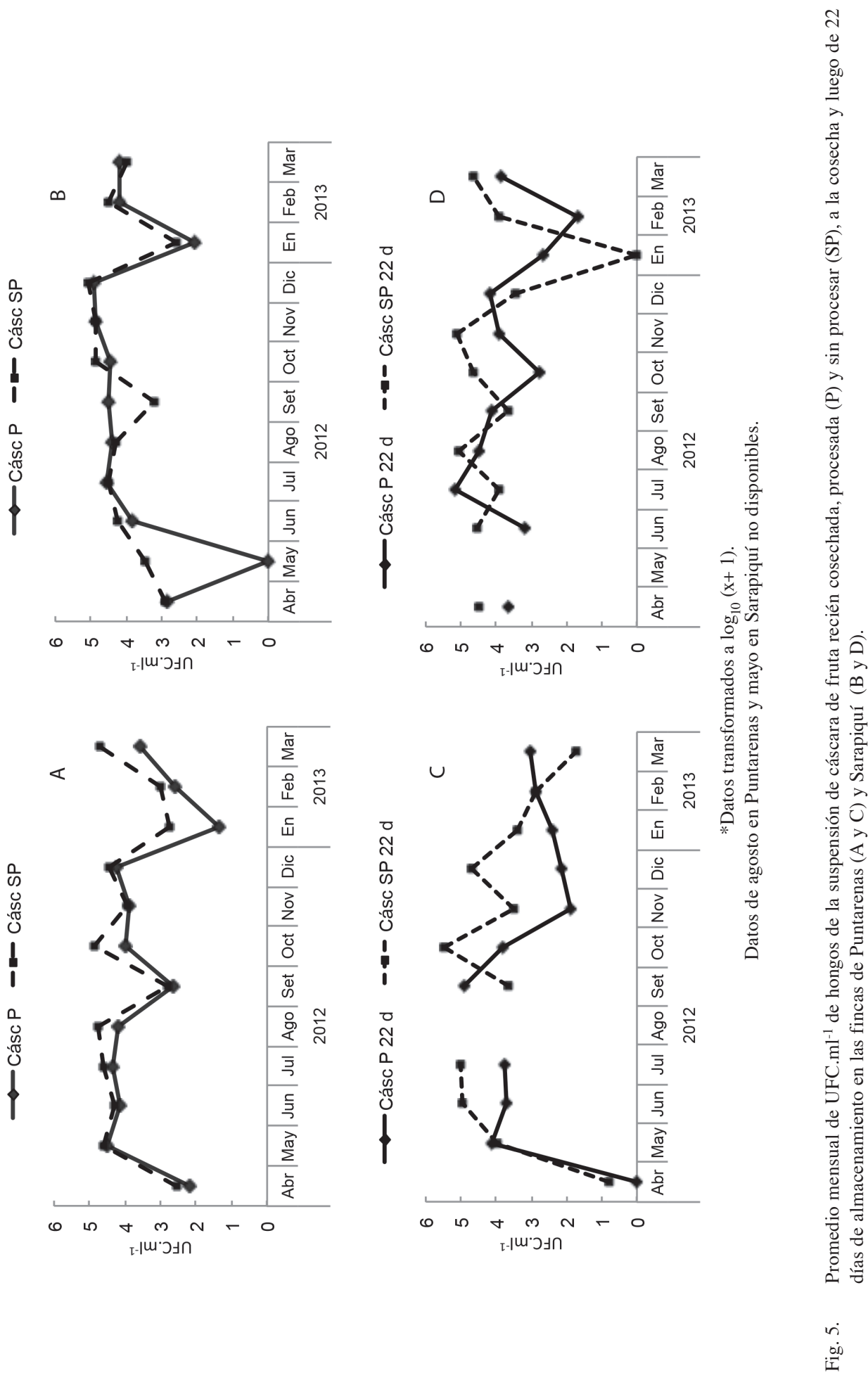

Agronomía Costarricense 39(3): 61-77. ISSN:0377-9424 / 2015 
La población de hongos en la cáscara de fruta recién cosechada (Figuras $5 \mathrm{~A}$ y $5 \mathrm{C}$ ) fue mayor que en el pedúnculo (Figura 3) en ambas zonas, y se observó esta misma tendencia durante todos los muestreos, tanto en fruta procesada como sin procesar.

La Figura 5B, correspondiente a las poblaciones en cáscara después del almacenamiento, muestra en Puntarenas épocas del año, mayo y junio de 2012 y de octubre de 2012 a febrero de 2013, en que la población de hongos en la cáscara de fruta procesada fue menor que en fruta sin procesar. En Sarapiquí (Figura 5D), no se presentó este mismo comportamiento sino que solamente en abril, junio, octubre y noviembre de 2012 y febrero y marzo de 2013, la población en fruta procesada fue menor que en fruta sin procesar.

En general, la población de hongos en la cáscara de fruta almacenada durante 22 días de Puntarenas (Figura 5B), tendió a ser menor en los meses de final e inicio de año, tal como se observa en los resultados obtenidos entre noviembre de 2012 y marzo de 2013 y en el primer muestreo de abril de 2012.

Las Figuras 6 y 7 muestran la incidencia y severidad de moho por mes en las fincas de Puntarenas y Sarapiquí. Se obtuvieron valores de incidencia cercanos o iguales al $100 \%$ en fruta sin procesar, durante la mayoría de los muestreos en ambas zonas, con excepción de abril de 2012 en Puntarenas y agosto y diciembre de 2012 en Sarapiquí.
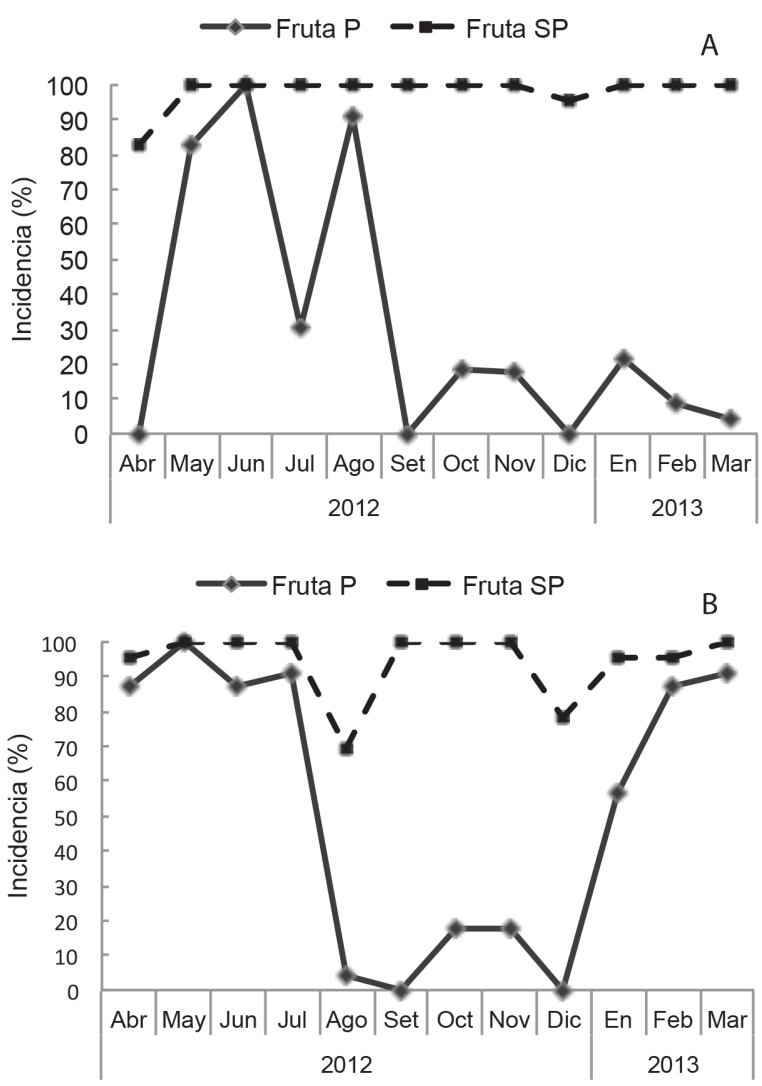

Fig. 6. Incidencia de moho en el pedúnculo de frutos de piña procesada (P) y sin procesar (SP) de las fincas de Puntarenas (A) y Sarapiquí (B), después de 22 días de almacenamiento. 

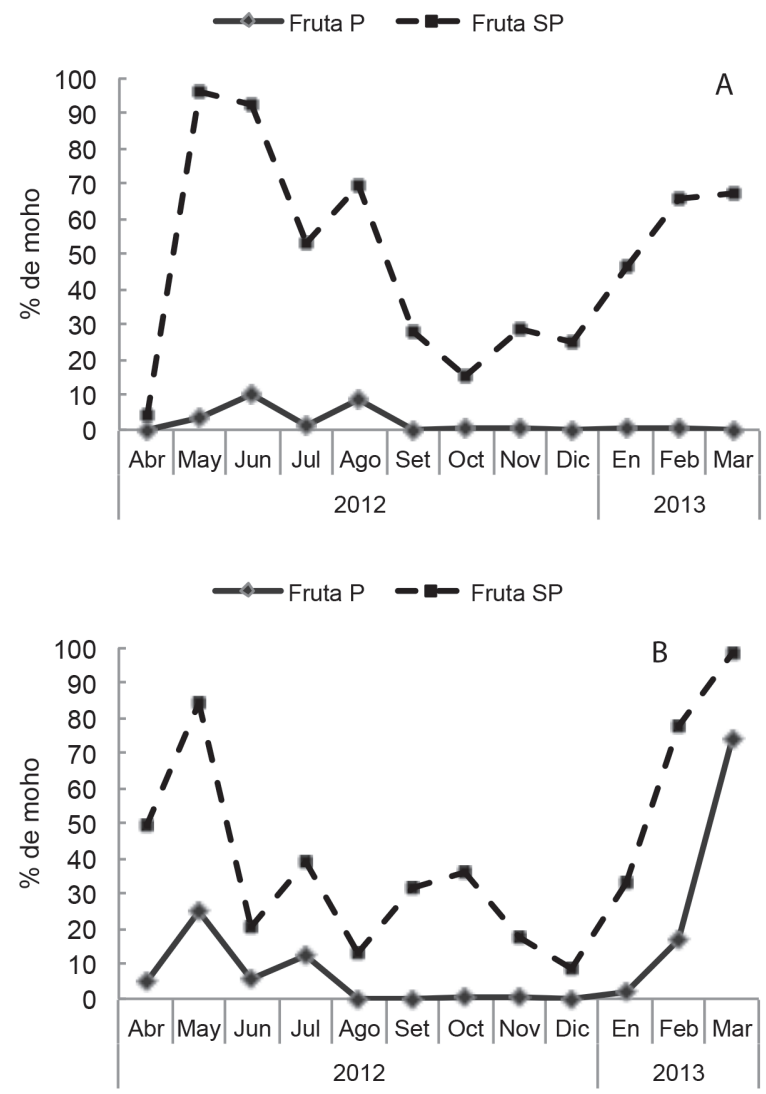

Fig. 7. Porcentaje de moho en el pedúnculo de frutos de piña procesada (P) y sin procesar (SP) de las fincas de Puntarenas (A) y Sarapiquí (B), después de 22 días de almacenamiento.

La mayor incidencia de moho en fruta procesada de Puntarenas se presentó de mayo a agosto de 2012, con valores entre $30 \%$ y $100 \%$, mientras que en Sarapiquí, la mayor incidencia se presentó de abril a julio de 2012 y febrero a marzo de 2013, con valores de aproximadamente $90 \%$. En las 2 zonas de estudio, en setiembre a diciembre de 2012 se obtuvieron valores bajos de incidencia en la fruta procesada con variaciones entre $0 \%$ y $20 \%$, comportamiento que se mantuvo en Puntarenas hasta marzo de 2013, mientras que en Sarapiquí tendió a aumentar a partir de enero y hasta marzo de 2013, donde se alcanzó un $90 \%$ (Figura 6).
El porcentaje de moho en el pedúnculo de la fruta sin procesar fue mayor que en fruta procesada en todos los meses para ambas zonas (Figura 7). En Puntarenas, el porcentaje máximo obtenido en fruta procesada fue del $10 \%$ en junio y agosto de 2012 y el resto del año se mantuvo entre $0,04 \%$ y $0,96 \%$, con excepción de abril, setiembre y diciembre de 2012, donde no hubo desarrollo de moho. A su vez, en Sarapiquí se obtuvo los mayores porcentajes de moho en fruta procesada, entre $5 \%$ y $25 \%$ de abril a julio de 2012, y en el 2013 se registró un aumento a partir de enero, hasta alcanzar un máximo de $75 \%$ en marzo. En el resto de los muestreos se obtuvo 
entre $0,04 \%$ y $0,48 \%$, con excepción de setiembre y diciembre de 2012, donde no hubo desarrollo de moho (Figura 7).

El análisis estadístico (Cuadro 1), muestra que en ambas fincas, la población de hongos en el pedúnculo de fruta recién cosechada no presentó diferencias significativas al comparar entre fruta procesada (P) y sin procesar (SP). Después de 22 días de almacenamiento, solamente en Puntarenas se obtuvo una población de hongos menor en fruta P que en SP. En la cáscara, solamente se obtuvo diferencias significativas en fruta recién cosechada, donde la población de hongos en Puntarenas fue mayor en fruta SP, que en fruta P.

Aunque en el análisis estadístico no se comparó los resultados entre zonas, el promedio de la población de hongos en el pedúnculo de fruta procesada fue mayor en Sarapiquí que en
Puntarenas, luego de 22 días de almacenamiento, y esto se reflejó en un mayor porcentaje de moho, donde se obtuvo un 11,6\% en Sarapiquí y en Puntarenas fue de $2,1 \%$.

En ambas fincas, la población de todos los microorganismos muestreados fue mayor en cera, que en aguas de lavado y desinfección con diferencias importantes, ya que en hongos se obtuvo como máximo 6,4 UFC.ml-1 en agua de Puntarenas, mientras que los promedios en cera fueron de 4.747,2 UFC. $\mathrm{ml}^{-1}$ en Puntarenas y 12.737,4 UFC. $\mathrm{ml}^{-1}$ en Sarapiquí.

En la Figura 8 se presenta la superficie de las colonias de los principales hongos recuperados al finalizar los 12 muestreos en cada zona. El principal género fue Penicillium, con 3 especies que desarrollaron colonias de color verde, con el borde redondeado y esporulación abundante

Cuadro 1. Comparación estadística entre los promedios de UFC de hongos en la fruta, agua y cera, y el porcentaje de moho en el pedúnculo de fruta procesada (P) y sin procesar (SP), de las fincas de Puntarenas y Sarapiquí.

\begin{tabular}{|c|c|c|c|c|c|c|}
\hline \multirow[b]{2}{*}{ Variables } & \multicolumn{3}{|c|}{ Puntarenas } & \multicolumn{3}{|c|}{ Sarapiquí } \\
\hline & Promedio & $\mathrm{P}(\mathrm{F})$ & $P(t)$ & Promedio & $\mathrm{P}(\mathrm{F})$ & $P(t)$ \\
\hline $\begin{array}{l}\text { UFC. } \mathrm{ml}^{-1} \text { pedúnculo } \mathrm{P} \text { cosecha } \\
\text { UFC. } \mathrm{ml}^{-1} \text { pedúnculo } \mathrm{SP} \text { cosecha }\end{array}$ & $\begin{array}{r}2.213,8 \mathrm{a} \\
950,3 \mathrm{a}\end{array}$ & $1,5 \times 10^{-19}$ & 0,52 & $\begin{array}{r}19,2 \mathrm{a} \\
707,3 \mathrm{a}\end{array}$ & 0,002 & 0,08 \\
\hline $\begin{array}{l}\text { UFC. } \mathrm{ml}^{-1} \text { pedúnculo } \mathrm{P} 22 \mathrm{~d} \\
\text { UFC. } \mathrm{ml}^{-1} \text { pedúnculo SP } 22 \mathrm{~d}\end{array}$ & $\begin{array}{l}16.181,8 \mathrm{~b} \\
76.727,3 \mathrm{a}\end{array}$ & $6,8 \times 10^{-9}$ & 0,0003 & $\begin{array}{l}100.318,2 \mathrm{a} \\
134.139,4 \mathrm{a}\end{array}$ & 0,35 & 0,33 \\
\hline $\begin{array}{l}\text { UFC. } \mathrm{ml}^{-1} \text { cásc } \mathrm{P} \text { cosecha } \\
\text { UFC. } \mathrm{ml}^{-1} \text { cásc } \mathrm{SP} \text { cosecha }\end{array}$ & $\begin{array}{l}28.638,3 \mathrm{~b} \\
72.514,3 \mathrm{a}\end{array}$ & $2,1 \times 10^{-8}$ & 0,02 & $\begin{array}{r}61.610,1 \mathrm{a} \\
97.504,3 \mathrm{a}\end{array}$ & $1,11 \times 10^{-5}$ & 0,12 \\
\hline $\begin{array}{l}\text { UFC. } \mathrm{ml}^{-1} \text { cásc P } 22 \mathrm{~d} \\
\text { UFC. } \mathrm{ml}^{-1} \text { cásc SP } 22 \mathrm{~d}\end{array}$ & $\begin{array}{l}211.454,6 \mathrm{a} \\
184.727,3 \mathrm{a}\end{array}$ & 0,008 & 0,84 & $\begin{array}{r}92.833,3 \mathrm{a} \\
166.000,0 \mathrm{a}\end{array}$ & $2,39 \times 10^{-9}$ & 0,15 \\
\hline $\begin{array}{l}\text { UFC.ml- }{ }^{-1} \text { agua } \\
\text { UFC. } \mathrm{ml}^{-1} \text { cera }\end{array}$ & $\begin{array}{r}6,4 \mathrm{~b} \\
4.747,2 \mathrm{a}\end{array}$ & 0 & $2,2 \times 10^{-9}$ & $\begin{array}{r}0,2 \mathrm{~b} \\
12.737,4 \mathrm{a}\end{array}$ & 0 & $2,3 \times 10^{-17}$ \\
\hline $\begin{array}{l}\% \text { moho fruta P } 22 \mathrm{~d} \\
\% \text { moho fruta SP } 22 \mathrm{~d}\end{array}$ & $\begin{array}{r}2,1 \mathrm{~b} \\
48,9 \mathrm{a}\end{array}$ & 0 & $3,2 \times 10^{-62}$ & $\begin{array}{l}11,6 \mathrm{~b} \\
42,5 \mathrm{a}\end{array}$ & $1,7 \times 10^{-13}$ & $1,5 \times 10^{-27}$ \\
\hline
\end{tabular}



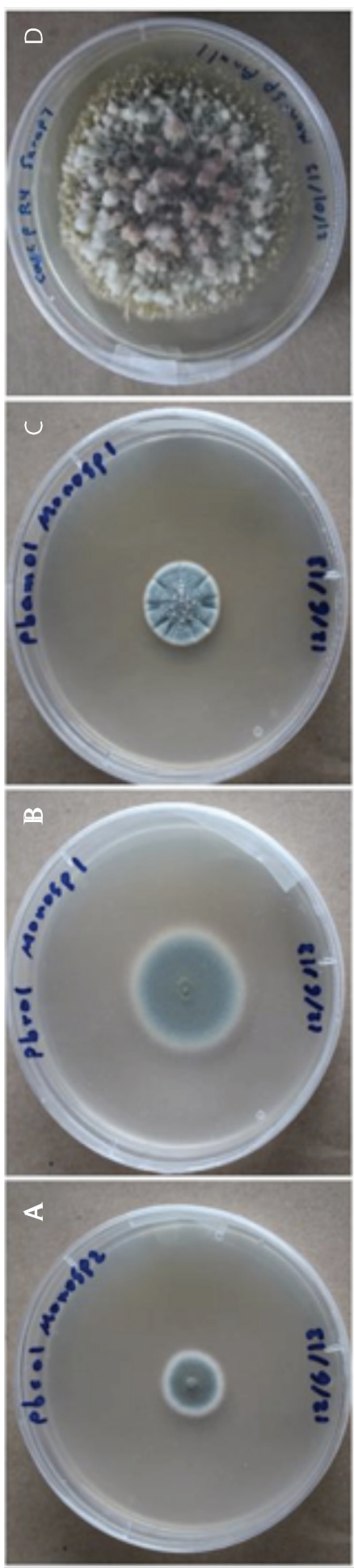
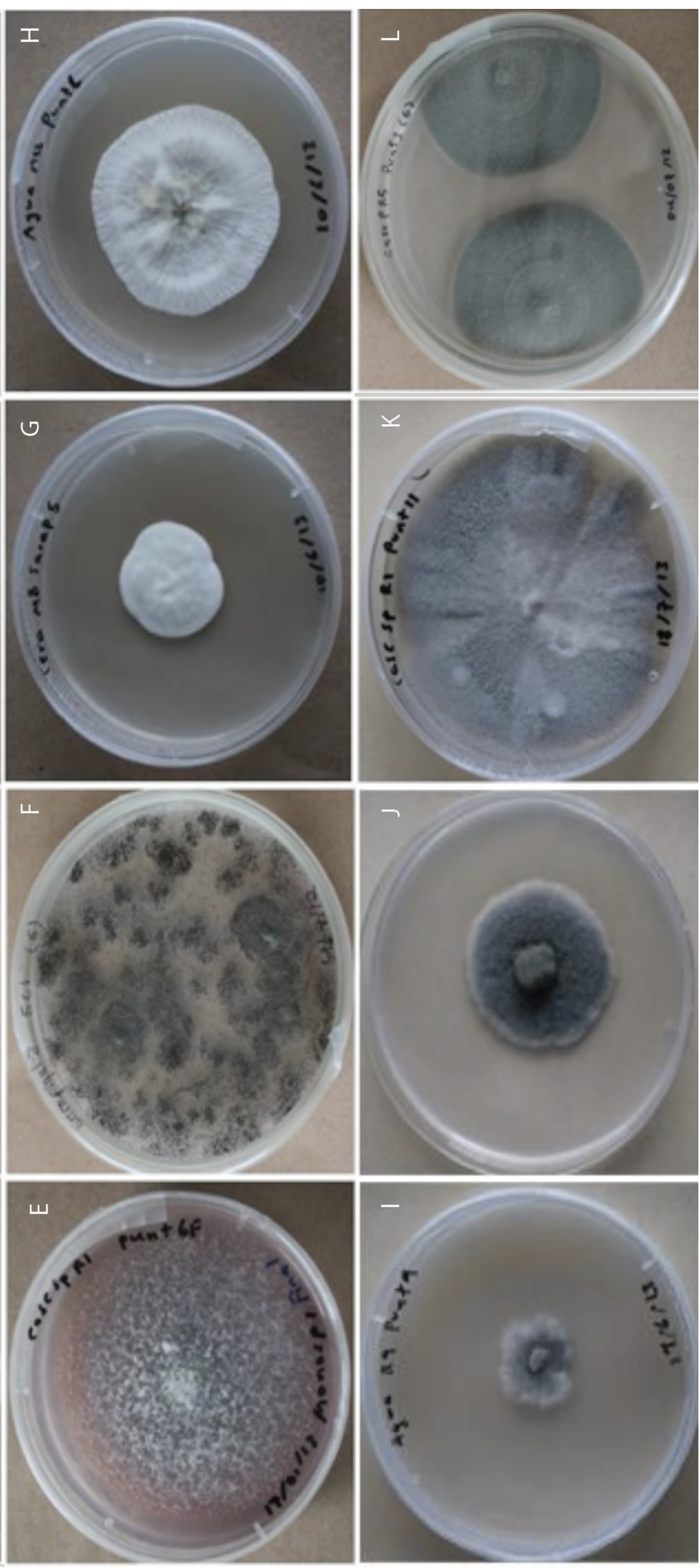

离

然

हิ

亲

:

ป

4 菏

원

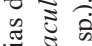

흥

年

$\frac{0}{0}$

0.

药

害

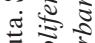

专

ㅍำ

व)

욜

皇

范

웅

유.

远

远

象

एँ

苛

政

范药

a $N$

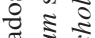

范 䨔

उ.

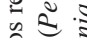

일

อิ

党

可范

言

范言

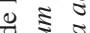

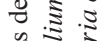

产

0ं 8

$\infty$ 
(Figura 8A, B y C). La especie Talaromyces calidicanius (Figura 8D), se caracterizó por un crecimiento de micelio color verde y rojizo en la parte aérea, con abundantes proyecciones superficiales de hifas y conidióforos que alcanzaron el borde superior de la placa Petri. Además, se recuperó Penicillium daleae, con una colonia de color blanco y poca esporulación (Figura 8G). El resto de microorganismos correspondió a hongos de micelio oscuro o blanco algodonoso, detallados a nivel de género o especie en la Figura 8.

\section{DISCUSIÓN}

El desarrollo de mohos en el pedúnculo de la fruta es un problema importante en la producción de piña para exportación como fruta fresca en Costa Rica (Barquero 2010), de ahí la importancia de conocer la dinámica de las poblaciones durante el año y los géneros presentes con mayor frecuencia para lograr establecer estrategias adecuadas de manejo. En el presente trabajo, las altas poblaciones de hongos recuperadas en el pedúnculo de la fruta, entre mayo y agosto de 2012 en Puntarenas y de abril a julio de 2012 en Sarapiquí, luego de 22 días de almacenamiento, son un buen parámetro para explicar la mayor incidencia y severidad obtenida en esos meses. Este mismo patrón se observó entre enero y marzo de 2013 en Sarapiquí, donde se obtuvo un incremento en las poblaciones de hongos en el pedúnculo de fruta procesada y sin procesar y como consecuencia de ello, también tendió a incrementar la incidencia y severidad de moho.

Las poblaciones de hongos que se obtuvieron en el pedúnculo de fruta recién cosechada, no reflejaron la tendencia en los valores de incidencia y severidad al finalizar el almacenamiento de la fruta, y un aspecto que pudo influir en este comportamiento fueron las especies asociadas y las diferencias en la velocidad de crecimiento de cada una (Castro 2015), lo que pudo permitir que, aunque inicialmente se encontraban en poblaciones relativamente bajas y no fueron recuperadas e identificadas con la metodología empleada, tuvieron la capacidad de crecer y esporular rápidamente en el pedúnculo y desarrollar cantidades importantes de moho, como respuesta a las prácticas poscosecha empleadas y a las condiciones de almacenamiento, como cambios en la humedad relativa y temperatura. Estas diferencias en la velocidad de crecimiento han sido informadas por otros autores como Baert et ál. (2008) y Morales et ál. (2008), quienes encontraron variaciones en la tasa de crecimiento de diferentes aislados de Penicillium expansum.

Las poblaciones de hongos en la cáscara de la fruta sin procesar cuando ingresó a la empacadora, se considera un mejor estimador que las poblaciones en el pedúnculo, ya que en ambas fincas, se registró en la cáscara poblaciones mayores a 1 UFC.ml-1 ${ }^{-1}$, detectándose los incrementos o disminuciones durante el año de estudio, y aunque no necesariamente los aumentos en las poblaciones de hongos en la cáscara coincidieron con una alta incidencia y severidad de moho al finalizar el almacenamiento de la fruta, este podría ser un parámetro útil para estimar la cantidad de inóculo que llega a la empacadora y entra en contacto con el agua de lavado y desinfección, la cera y las diferentes superficies con las que tiene contacto la fruta durante su procesamiento poscosecha, tal como lo mencionan Barth et ál. (2009) y Prusky y Gullino (2010).

La diferencia observada entre las poblaciones de hongos en el pedúnculo de fruta procesada y sin procesar, muestra el efecto positivo del procesamiento poscosecha empleado en ambas empacadoras, en la reducción de microorganismos en esa zona. Al igual que en otras empacadoras de piña de Costa Rica y otros países, la desinfección de la fruta en agua con concentraciones de cloro entre 50 y 150 ppm y el uso de fungicidas poscosecha aplicados directamente al pedúnculo, son prácticas comunes (MAG 2010, García y Rodríguez 2011, Hu et ál. 2011, Paull y Duarte 2011) y que pudieron influir en las diferencias encontradas durante la mayoría de los muestreos, donde se obtuvo las poblaciones más altas de microorganismos y desarrollo de moho en la fruta que no recibió estos tratamientos poscosecha, sin embargo, aún cuando hubo una 
reducción de la severidad del moho, no se evitó por completo su crecimiento, lo cual muestra la necesidad de buscar alternativas de manejo para lograr un mejor control del desarrollo de moho en el pedúnculo.

En comparación con el pedúnculo, en la cáscara la diferencia en las poblaciones de hongos entre fruta procesada y sin procesar fue menor, lo cual pudo deberse a que durante el proceso poscosecha, la aplicación de fungicida se realizó únicamente al pedúnculo; además, estos resultados mostraron que la desinfección con cloro no redujo las poblaciones de microorganismos en la cáscara lo suficiente, las cuales pudieron continuar multiplicándose y se mantuvieron en este tejido, hasta finalizar el período de almacenamiento, sin embargo, no fue evidente el desarrollo de moho en este tejido, mientras que en el pedúnculo, con poblaciones iguales o menores que las de cáscara, se obtuvo un crecimiento importante de moho, lo cual indica que este tejido proveé mejores condiciones para el desarrollo de micelio que la cáscara, posiblemente debido a que el corte del pedúnculo es una herida en la fruta, a partir de la cual pueden fluir sustancias nutritivas, entre estas los azúcares (Paull y Reyes 1996) y que sirven como sustrato para diversos microorganismos. Además, la cáscara de la piña y otros frutos presenta un mayor contenido de sustancias como polifenoles, cutina y ceras, que reducen el crecimiento de microorganismos y protegen la fruta de infecciones por diversos patógenos (Bocco et ál. 1998, Guo et ál. 2003, Mokbel y Hashinaga 2005, Lata et ál. 2009, Chanda et ál. 2010, Lara et ál. 2014), lo cual pudo influir significativamente para que aún con las poblaciones recuperadas, no se obtuviese desarrollo de moho en ese tejido.

Las bajas poblaciones de hongos en el agua de lavado y desinfección, podrían deberse a las concentraciones de cloro en el agua de las pilas, tratamiento comúnmente utilizado en piña (MAG 2010, García y Rodríguez 2011), que en el presente estudio se empleó en concentraciones entre 150 a $180 \mathrm{ppm}$ de cloro libre durante todos los muestreos.
Como indican Arauz (1994), Araya y Cascante (2000) y Hui et ál. (2006), el aire, el agua de lavado y desinfección y cada una de las superficies con que entra en contacto la fruta pueden ser una fuente de inóculo de los patógenos que causan el desarrollo de mohos y pudriciones poscosecha. Al comparar las poblaciones de hongos en el agua de lavado y desinfección y en la cera (Figura 1), se observó que la cera constituye una fuente mayor de inóculo para el pedúnculo de la fruta en comparación con el agua, esto posiblemente debido a que el sistema de aplicación de las ceras en piña requiere de una recirculación de la misma, debido al costo económico de este insumo, lo que podría favorecer que con el transcurso del proceso, se acumulen propágulos de diseminación que podrían quedar depositados en el pedúnculo durante su aplicación. Además, las poblaciones en la cera (Cuadro 1), fueron mayores a 1000 UFC, determinado por López (2012), como la cantidad mínima para iniciar el crecimiento de moho en el pedúnculo, causado por los géneros Trichoderma, Penicillium, Aspergillus y Fusarium, en fruta inoculada, lo cual incrementa el riesgo de desarrollo de moho en el pedúnculo, al recircular la cera y aplicarla directamente sobre la fruta.

En el aire de las cámaras de enfriamiento y almacenamiento se recuperaron esporas de hongos que por el mecanismo de recirculación de ese aire, podrían alcanzar la superficie del pedúnculo y dependiendo de la especie presente, contribuir al desarrollo de mohos. Además, la obtención de poblaciones de hongos más altas durante todos los muestreos en la zona de Sarapiquí, en comparación con Puntarenas, podría deberse a diferencias en la eficacia de los protocolos de limpieza de las cámaras de ambas fincas y a que en Sarapiquí se procesó mayores volúmenes de fruta durante el año, lo cual pudo aumentar la cantidad de esporas de hongos en el aire de las cámaras.

Morales et ál. (2008 y 2010) informan que con la adecuada limpieza y desinfección de las plantas empacadoras y de las cámaras de enfriamiento y almacenamiento de manzana, se logró disminuir significativamente el nivel de 
inóculo y la incidencia de fruta con moho causado por Penicillium expansum. Además, trabajos realizados en este mismo cultivo por Amiri y Bompeix (2005), mostraron una relación directa entre la cantidad de esporas por $\mathrm{m}^{3}$ en el aire de las cámaras de almacenamiento y la densidad de esporas del género Penicillium en la superficie de frutos de manzana, por lo tanto, aunque no fue una de las variables medidas en el presente estudio, la adecuada limpieza y desinfección de las instalaciones y equipo utilizado en la planta empacadora y de las cámaras de enfriamiento en piña, son aspectos que deberían tomarse en cuenta para disminuir la posibilidad de que posterior a la aplicación de cloro y fungicida, lleguen esporas de hongos al pedúnculo, que podrían originar el desarrollo de mohos en poscosecha.

El hongo más frecuente en el presente trabajo correspondió al género Penicillium, con 4 especies diferentes, de las cuales $P$. purpureogenum, P. diversum y Penicillium sp., presentaron abundante esporulación en medio de cultivo PDA $+\mathrm{Al}$ y predominio de crecimiento en el pedúnculo de los frutos de ambas zonas y por lo tanto, disminuir la llegada de estas especies al pedúnculo de la piña, debe ser uno de los principales objetivos si se desea logar un manejo eficiente de los mohos poscosecha en este tejido. Penicillium es uno de los géneros más importantes citados como patógenos causantes de mohos en piña (Malins 1991, Hui et ál. 2006, Mitra 1997). Mourichon (1998) y Bartholomew et ál. (2003) citan que Penicillium funiculosum se encuentra directamente asociado con la enfermedad conocida como pudrición de los frutículos, lo que evidencia que es un microorganismo importante en este cultivo.

De las demás especies identificadas, solamente se encontró información de los géneros Fusarium y Aspergillus, citados como patógenos importantes en piña (Snowdon 2000, López 2012, Jacobs et ál. 2010). De estos, se ha informado que Fusarium tiene la capacidad de colonizar los diferentes tejidos de la planta de piña (Snowdon 2000, Jacobs et ál. 2010, Stępień et ál. 2011a, Stępień et ál. 2011b, Stępień et ál. 2013), sin embargo, su velocidad de crecimiento es menor que la de Penicillium (Castro 2015), lo cual muestra diferencias importantes en el potencial que ambos microorganismos tienen para desarrollar moho en el pedúnculo. Los hongos de los géneros Phoma y Cladosporium han sido señalados como patógenos en diversos cultivos tropicales como papaya, banano y mango (Alvarez y Nishijima 1987, Snowdon 2000, CAB 2002, Anthony et ál. 2004) y debido a sus características de abundante esporulación y crecimiento de micelio de color oscuro, son microorganismos que pueden contribuir al desarrollo de moho poscosecha en el pedúnculo de la piña.

Los resultados obtenidos en el presente trabajo mostraron variaciones importantes en las poblaciones de hongos, incidencia y severidad de los mohos en el pedúnculo de la piña durante el año en ambas zonas de estudio, razón por la cual se considera importante el monitoreo constante de las poblaciones y los géneros de hongos presentes en la fruta durante las diferentes fases poscosecha, evaluando la posibilidad de generar umbrales para cada especie en particular, o bien, de utilizar técnicas como el PCR en tiempo real, de manera que se logre una rápida identificación de los patógenos presentes e implementar y dar seguimiento a los protocolos ya establecidos de limpieza y desinfección de las instalaciones y equipo de la planta empacadora y de las cámaras de almacenamiento, con mayor cuidado en esas épocas, para disminuir el desarrollo de mohos en el mercado destino.

Debido a que se encontró que la cera acumula cargas significativas de hongos durante el procesamiento de la fruta, se considera importante la búsqueda de opciones para evitar el uso de la cera recirculada, como podría ser el estudio de otras formas de aplicación como la aspersión de la cera a la fruta, o bien explorar la posibilidad de adicionar sustancias GRAS (Generally recognized as safe, por sus siglas en inglés), con propiedades fungistáticas o fungicidas o bien, investigar el efecto de la pasteurización de la cera o algún otro tratamiento físico todo con el objetivo de 
que no se acumulen poblaciones importantes de microorganismos en ese tratamiento.

Así mismo, las diferencias en la incidencia y severidad de moho entre fruta procesada y sin procesar, son un indicador de que, el adecuado cumplimiento de las labores poscosecha comúnmente utilizadas en piña y el almacenamiento a baja temperatura, tienen un impacto positivo en la reducción de los mohos en el pedúnculo, aunque no siempre son suficientes para evitar por completo el desarrollo de los mismos.

\section{AGRADECIMIENTOS}

A los Sres. Francisco López y Eduardo Naranjo por las facilidades brindadas durante el planteamiento y la ejecución de los muestreos en las empacadoras.

\section{LITERATURA CITADA}

ALVAREZ A.M., NISHIJIMA W.T. 1987. Postharvest diseases of papaya. Plant Disease 71(8):681-686.

AMIRI A., BOMPEIX G. 2005. Diversity and population dynamics of Penicillium spp., on apples in pre-and postharvest environments: consequences for decay development. Plant Pathology 54:74-81.

ANTHONY S., ABEYWICKRAMA K., DAYANANDA R., WILSON S., ARAMBEWELA L. 2004. Fungal pathogens associated with banana fruit in Sri Lanka and their treatment with essential oils. Mycopathologia 157:91-97.

ARAUZ F. 1994. Elementos básicos de patologia poscosecha de frutas y hortalizas. I Taller regional de manejo poscosecha de productos de interés para el Trópico. San José, Costa Rica. pp. 1-10.

ARAYA B., CASCANTE M. 2000. Manejo post-cosecha de productos agrícolas. San José, Costa Rica. Editorial UNED. 219 p.

BAERT K., DEVLIEGHERE F., BO L., DEBEVERE J., DE MEULENAER B. 2008. The effect of inoculum size on the growth of Penicillium expansum in apples. Food Microbiology 25:212-217.

BARNETT H.L., HUNTER B.B. 1972. Illustrated genera of imperfect fungi. Third edition. Minnesota, USA. Burguess Publishing Company. $241 \mathrm{p}$.

BARQUERO A. 2010. Estudio comparativo de la eficacia de cinco desinfectantes y optimización del mejor de ellos para la etapa de desinfección de piña fresca en la empresa Banacol. Práctica Dirigida Escuela de Tecnología de Alimentos para optar por el grado de licenciatura en Tecnología de Alimentos. Costa Rica, Universidad de Costa Rica. 57 p.
BARTH M., HANKINSON T., ZHUANG H., BREIDT F. 2009. Microbiological spoilage of fruits and vegetables. In: W. Sperber and M. Doyle (eds.). Compendium of the microbiological spoilage of foods and beverages. New York, USA. Springer. $367 \mathrm{p}$

BARTHOLOMEW D.P., PAULL R.E., ROHRBACH K.G. 2003. The pineapple, botany, production and uses. New York, USA. CABI Publishing. 301 p.

BOCCO A., CUVELIER M., RICHARD H., BERSET C 1998. Antioxidant activity and phenolic composition of citrus peel and seed extracts. Journal of Agriculture and Food Chemistry 46:2123-2129.

BUTTNER M.P., STETZENBACH L.D. 1993. Monitoring airborne fungal spores in an experimental indoor environment to evaluate sampling methods and the effects of human activity on air sampling. Applied and Environmental Microbiology 59(1):219-226.

CAB. 2002. Phoma herbarum. In: Descriptions of fungi and bacteria. CABI. 151: Sheet 1501. Consultado el 25 de noviembre de 2013. Disponible en www.cabi.org/dfb

CANAPEP 2015. Estadísticas de exportaciones Costa Rica. Consultado el 25 de febrero de 2015. Disponible en www.canapep.com

CASTRO J. 2015. Hongos causantes de mohos en frutos de piña poscosecha, su incidencia y relación con las condiciones climáticas en dos zonas de Costa Rica. Tesis de maestría, Universidad de Costa Rica. Costa Rica. $111 \mathrm{p}$.

CHANDA S., BARAVALIA Y., KANERIA M., RAKHOLIYA K. 2010. Fruit and vegetable peelsstrong natural source of antimicrobics, pp. 444-450. In: M. Vilas, (ed.). Current Research Technology and Education Topics in Applied Microbiology and Microbial Biotechnology. Badajoz, España.

DENNIS C. 1983. Post-harvest pathology of fruits and vegetables. London, England. Academic Press. $264 \mathrm{p}$.

ELLIS M.B. 1976. More dematiaceous hyphomycetes. Surrey, England. CAB. 507 p.

FRENCH E., HEBERT T. 1980. Métodos de investigación fitopatológica. San José, Costa Rica. IICA. 289 p.

GARCÍA A., RODRÍGUEZ M. 2011. Manual de buenas prácticas agrícolas para la producción de piña en Costa Rica. San José, Costa Rica. Banacol-RepCar. $66 \mathrm{p}$.

GUO C., YANG J., WEI J., LI Y., XU J., JIANG Y. 2003. Antioxidant activities of peel, pulp and seed fractions of common fruits as determined by FRAP assay. Nutrition Research 23:1719-1726.

HU H., LI X., DONG C., CHEN W. 2011. Effects of wax treatment on quality and postharvest physiology of pineapple fruit in cold storage. African Journal of Biotechnology 10(39):7592-7603.

HUI Y., BARTA J., CANO M., GUSEK T. 2006. Handbook of fruits and fruit processing: science and technology. Iowa, USA. Blackwell Publishing. 697 p. Consultado el 30 de setiembre de 2011. Disponible en http:// books.google.com 
JACOBS A., VAN WYK P.S., MARASAS W.F., WINGFIELD B.D., WINGFIELD M.J., COUTINHO T.A. 2010. Fusarium ananatum sp. Nov in the Giberella fujikuroi species complex from pineapples with fruit rot in South Africa. Fungal Biology 114:515-527.

LARA I., BELGE B., GOULAO L.F. 2014. The fruit cuticle as a modulator of postharvest quality. Postharvest Biology and Technology 87:103-112.

LATA B., TRAMPCZYNSKA A., PACZESNA J. 2009. Cultivar variation in apple peel and whole fruit phenolic composition. Scientia Horticulturae 121:176-181.

LÓPEZ C. 2012. Frecuencia de los principales organismos asociados con el moho en el corte de piña (Ananas comosus) Var. Dorada Extradulce, patogenicidad y sensibilidad al fungicida triadimefon. Tesis licenciatura en Agronomía. Costa Rica. Universidad de Costa Rica. 71 p.

MAG. 2010. Manual de buenas prácticas agrícolas para la producción de piña. Heredia, Costa Rica. MAG, SFE. $133 \mathrm{p}$.

MALINS A. 1991. Second regional workshop in tropical fruit crops, papaya, pineapple and mango. Antigua \& Barbuda. IICA. 97 p.

MITRA S. 1997. Postharvest physiology and storage of tropical and subtropical fruits. London, England. Cab International. $423 \mathrm{p}$.

MOKBEL M.S., HASHINAGA F. 2005. Antibacterial and antioxidant activities of banana peel (Musa AAA cv. Cavendish) fruits peel. American Journal of Biochemistry and Biotechnology 1(3):125-131.

MONTERO M., ROJAS M.A., MARTÍN O. 2008. Effect of packaging conditions on quality and shelf-life of fresh-cut pineapple (Ananas comosus). Postharvest Biology and Technology 50:182-189.

MORALES H., MARÍN S., RAMOS A., SANCHIS V. 2010. Influence of post-harvest technologies applied during cold storage of apples in Penicillium expansum growth and patulin accumulation: A review. Food control 21:953-962.

MORALES H., SANCHIS V., COROMINES J., RAMOS A., MARÍN S. 2008. Inoculum size and intraspecific interactions affects Penicillium expansum growth and patulin accumulation in apples. Food Microbiology 25:378-385.

MOURICHON X. 1998. Pineapple fruit core rot (black spot) and leathery pocket: review and prospects. Acta Horticulturae, Proceedings of $2^{\text {nd }}$ International Pineapple Symposium. Montpellier, Francia. 508 p.

PAULL E., DUARTE O. 2011. Tropical fruits. London, England. Cab International. $2^{\text {nd }}$ Edition. 400 p.
PAULL R.E., REYES M.E. 1996. Preharvest weather conditions and pineapple fruit translucency. Scientia Horticulturae 66:59-67.

PITT J., HOCKING A.D. 2009. Fungi and food spoilage. New York, USA. Springer. 519 p. Consultado el 10 de marzo de 2015. Disponible en books.google. co.cr/books

PROCOMER. 2015. Portal estadístico de comercio exterior. Consultado el 25 de febrero de 2015. Disponible en www.procomer.com

PRUSKY D., GULLINO M. 2010. Postharvest Pathology. New York, USA. Springer. $1150 \mathrm{p}$.

REYES D. 2012. Compuestos GRAS para el control de patógenos poscosecha in vitro en mango (Mangifera indica L.), piña (Ananas comosus L.) y papaya (Carica papaya L.), y pruebas de eficacia in vivo en piña. Tesis de licenciatura, Universidad de Costa Rica, Costa Rica. 92 p.

REYES M. 1999. Use of microbial antagonists to control postharvest black rot on pineapple fruit. Tesis de doctorado, Universidad de Hawai, Hawai.128 p.

SEIFERT K., MORGAN G., GAMS W., KENDRICK B. 2011. The genera of hyphomycetes. Utrecht, Netherlands. CBS-KNAW Fungal Biodiversity Centre. 997 p.

SNOWDON A. 2000. A color Atlas of post-harvest diseases $\&$ disorders of fruits and vegetables. Vol 1. Florida, USA. CRC Press. 302 p.

STĘPIEŃ L., KOCZYK G., WAŚKIEWICZ A. 2011a. FUM cluster divergence in fumonisins-producing Fusarium species. Fungal Biology 115:112-123.

STĘPIEŃ L., KOCZYK G., WAŚKIEWICZ A. 2011b. Genetic and phenotypic variation of Fusarium proliferatum isolates from different host species. Journal of Applied Genetics 52:487-496.

STĘPIEŃ L., KOCZYK G., WAŚKIEWICZ A. 2013. Diversity of Fusarium species and mycotoxins contaminating pineapple. Journal of Applied Genetics 54(3):367-380.

TORTORA G., FUNKE B., CASE C. 2007. Introducción a la microbiología. Buenos Aires, Argentina. Editorial Médica Panamericana. $9^{\circ}$ Edición. 963 p.

VON ARX J.A. 1974. The genera of fungi. Sporulating in pure culture. Leutershausen, Germany. J. Cramer. $315 \mathrm{p}$.

WATANABE T. 1994. Soil and seed fungi. Morphologies of cultured fungi and key to species. Tokyo, Japan. Lewis Publishers. $411 \mathrm{p}$.

WILLS R., McGLASSON B., GRAHAM D., JOYCE D 1998. Postharvest. An introduction to the physiology \& handling of fruit, vegetables \& ornamentals. Sydney, Australia. Cab International.1 ed. 262 p.

Todos los derechos reservados. Universidad de Costa Rica. Este artículo se encuentra licenciado con Creative Commons Reconocimiento-NoComercial-SinObraDerivada 3.0 Costa Rica. Para mayor información escribir a rac.cia@ucr.ac.cr 
